\section{Band 17, Heft 1, Februar 2010}

\section{Editorial}

4 «Integrative Medizin»- die Kolonialisierung des Anderen und die Notwendigkeit des ganz Anderen Walach, H. (Frankfurt/O.)

\section{Originalarbeiten}

7 Hypericumextrakt beim depressiven Syndrom: Eine offene Studie bei ambulanten Patienten Melzer, J. (Zürich, Oetwil am See); Brignoli, R. (Rüschlikon); Keck, M. (Oetwil am See); Saller, R. (Zürich)

15 Vergleichende Untersuchungen zur AntiHerpeswirkung von wässrigen und alkoholischen Extrakten von Cajanus cajan (L.) Millsp. Zu, Y.; Fu, Y.; Wang, W.; Wu, N.; Liu, W.; Kong, Y. (Harbin); Schiebel, H.M. (Braunschweig); Schwarz, G. (Harbin); Schnitzler, P.; Reichling, J. (Heidelberg)

21 Akupunktur induziert eine pro-inflammatorische Immunantwort verstärkt durch einen Konditionierungs-Erwartungs-Effekt Karst, M.; Schneidewind, D.; Scheinichen, D.; Juettner, B.; Bernateck, M. (Hannover); Molsberger, A. (Düsseldorf); Parlesak, A. (Kgs. Lyngby); Passie, T.; Hoy, L.; Fink, M. (Hannover)

Kurzmitteilung

29 Die mögliche Rolle von Thymian bei der Behandlung der Pyodermie im Neugeborenenalter eine retrospektive Analyse Thiel, M.T.; Sitzmann, F.; Längler, A. (Herdecke)

Briefe an die Herausgeber

32 Zu: Milgrom LR: Under Pressure: Homeopathy and Its Detractors. Forsch Komplementmed 2009;16:256-261 Ernst, E. (Exeter)

33 Zu: Michalsen A: Ernährungstherapie: Königin oder Aschenputtel der Naturheilkunde und Komplementärmedizin? Forsch Komplementmed 2009;16:144-145

Arends, J. (Freiburg i.Br.)

\section{Vol. 17, Issue 1, February 2010}

\section{Editorial}

4 'Integrative Medicine' - Colonialisation of the Other, and the Necessity of the Totally Other Walach, H. (Frankfurt/O.)

\section{Original Articles}

7 A Hypericum Extract in the Treatment of Depressive Symptoms: An Open Study in Outpatients Melzer, J. (Zürich, Oetwil am See); Brignoli, R. (Rüschlikon); Keck, M. (Oetwil am See); Saller, R. (Zürich)

15 Comparative Study on the Antiherpetic Activity of Aqueous and Ethanolic Extracts Derived from Cajanus cajan (L.) Millsp. Zu, Y.; Fu, Y.; Wang, W.; Wu, N.; Liu, W.; Kong, Y. (Harbin); Schiebel, H.M. (Braunschweig); Schwarz, G. (Harbin); Schnitzler, P.; Reichling, J. (Heidelberg)

21 Acupuncture Induces a Pro-Inflammatory Immune Response Intensified by a Conditioning-Expectation Effect

Karst, M.; Schneidewind, D.; Scheinichen, D.; Juettner, B.; Bernateck, M. (Hannover); Molsberger, A. (Düsseldorf); Parlesak, A. (Kgs. Lyngby); Passie, T.; Hoy, L.; Fink, M. (Hannover)

\section{Short Communication}

29 The Potential Role of Thyme in the Treatment of Pyodermia in Newborn Infants - a Retrospective Analysis Thiel, M.T.; Sitzmann, F.; Längler, A. (Herdecke)

Letters to the Editors

32 On: Milgrom LR: Under Pressure: Homeopathy and Its Detractors. Forsch Komplementmed 2009;16:256-261 Ernst, E. (Exeter)

33 On: Michalsen A: Ernährungstherapie: Königin oder Aschenputtel der Naturheilkunde und Komplementärmedizin? Forsch Komplementmed 2009;16:144-145

Arends, J. (Freiburg i.Br.)

Peer Review: Each manuscript is evaluated by the editors and by externa peer reviewers.

\section{KARGER}

Fax +497614520714

Information@Karger.de

www.karger.com 


\section{Band 17, Heft 1, Februar 2010}

Kongressbericht

34 Integrative Medizin weiter etabliert Dylus, J. (Weimar)

35 Journal Club Melchart, D.; Weidenhammer, W. (München)

35 Die Kunst der Integration in der allgemeinärztlichen Versorgung

36 Eigenbluttherapie bei akuten grippalen Infekten wenig überzeugend

37 CAM als Türöffner zur Gesundheitsförderung

39 Gebrauchsfertiges Gewichtsstabilisierungsprogramm

Abstract Service

41 Latest Publications You Shoud Not Miss

Gesellschaftsmitteilungen

43 Schweizerische Medizinische Gesellschaft für Phytotherapie (SMGP)

\section{Buchbesprechung}

48 Innovationen

50 News / Ticker

51 Fort- und Weiterbildung

52 Tagungen und Kongresse

54 Hinweise für Autoren

27 Impressum
Vol. 17, Issue 1, February 2010

Congress Report

34 Integrative Medicine further Established Dylus, J. (Weimar)

35 Journal Club Melchart, D.; Weidenhammer, W. (München)

35 The Art of Integrating in Practical Care

36 Autologous Blood Therapy for Acute Inspiratory Tract Infections Little Convincing

37 CAM as a Door Opener to Health Promotion

39 Ready-to-Use Weight Maintenance Program

Abstract Service

41 Latest Publications You Shoud Not Miss

Society Bulletins

43 Schweizerische Medizinische Gesellschaft für Phytotherapie (SMGP)

$\begin{array}{ll}\mathbf{4 6} & \text { Book Review } \\ \mathbf{4 8} & \text { Innovations } \\ \mathbf{5 0} & \text { News / Ticker } \\ \mathbf{5 1} & \text { Education } \\ \mathbf{5 2} & \text { Meetings and Conferences } \\ \mathbf{5 5} & \text { Guidelines for Authors } \\ \mathbf{2 7} & \text { Imprint }\end{array}$

Forthcoming papers are listed on page 56. 